\title{
Chronic hepatitis infection is associated with extrahepatic cancer development: a nationwide population-based study in Taiwan
}

Abram Bunya Kamiza ${ }^{1}$, Fu-Hsiung Su, 2,3,4, Wen-Chang Wang ${ }^{6}$, Fung-Chang Sung ${ }^{7,8}$, Shih-Ni Chang ${ }^{7,8}$ and Chih-Ching Yeh ${ }^{1,9^{*}}$

\begin{abstract}
Background: Hepatitis B virus (HBV) and hepatitis C virus (HCV) are the major causes of chronic hepatitis infection $(\mathrm{CHI})$. This longitudinal cohort study investigated the association of $\mathrm{CHI}$ with hepatic and extrahepatic cancer development in Taiwan.

Methods: Patients with HBV infection and HCV infection were identified from the Taiwan National Health Insurance Research Database. A Cox proportional hazard model was used to calculate hazard ratios (HRs) and $95 \%$ confidence intervals $(\mathrm{Cls})$ for determining the association between $\mathrm{CHI}$ and cancer development.

Results: The patients with HBV infection exhibited an increased risk of colorectal cancer (HR: 1.36, 95 \% Cl: 1.09-1.70), liver cancer (HR: 21.47, 95 \% Cl: 18.0-25.6), gallbladder and extrahepatic bile duct cancer (HR: 2.05, 95 \% Cl: 1.07-3.91), pancreatic cancer (HR: 2.61, 95 \% Cl: 1.47-4.61), kidney cancer (HR: 1.72, 95 \% Cl: 1.10-2.68), ovarian cancer (HR: 2.31, $95 \%$ Cl: 1.21-4.39), and non-Hodgkin's lymphoma (HR: 2.10, 95 \% Cl: 1.25-3.52). The patients with HCV infection exhibited an increased risk of liver cancer (HR: 25.10, $95 \%$ Cl: 20.9-30.2), gallbladder and extrahepatic bile duct cancer (HR: 2.60, 95 \% Cl: 1.42-4.73), ovarian cancer (HR: 5.15, 95 \% Cl: 1.98-13.4), and non-Hodgkin's lymphoma (HR: 2.30, $95 \%$ Cl: 1.34-3.96).
\end{abstract}

Conclusion: The present population-based study revealed that in addition to its association with primary liver cancer, $\mathrm{CHI}$ is associated with an increased risk of extrahepatic cancer.

Keywords: Hepatitis B virus, Hepatitis C virus, Cancer risk, Taiwan

\section{Background}

Hepatitis B virus (HBV) and hepatitis C virus (HCV) are the major causes of chronic hepatitis infection (CHI). Approximately 2 billion people worldwide have been infected with HBV, and 360 million people are currently chronic carriers [1]. HCV has been estimated to infect approximately 185 million people worldwide, with the highest prevalence in Central and East Asian, North African, and Middle Eastern regions [2], and more than

\footnotetext{
* Correspondence: ccyeh@tmu.edu.tw

'School of Public Health, College of Public Health and Nutrition, Taipei

Medical University, No. 250 Wu-Hsing Street, Taipei 11031, Taiwan

${ }^{9}$ Department of Public Health, China Medical University, No. 91 Hsueh-Shih

Road, Taichung 40402, Taiwan

Full list of author information is available at the end of the article
}

$75 \%$ of chronic HBV carriers reside in Asian countries, including Taiwan [3]. The prevalence of hepatitis B surface antigen (HBsAg) carriers in Asia is estimated to be $8-12 \%$ [4]. Patients with $\mathrm{CHI}$ are at an increased risk of liver fibrosis, liver cirrhosis, and hepatocellular carcinoma $[5,6]$.

Epidemiological studies have reported an association between $\mathrm{CHI}$ and primary liver cancer development [6-9]. Furthermore, some studies have revealed an association between $\mathrm{CHI}$ and the development of extrahepatic cancers such as pancreatic cancer [10], gallbladder and extrahepatic bile duct cancer [11], intrahepatic cholangiocarcinoma, and non-Hodgkin's lymphoma [12-15]. A study in 
Sweden reported an association between chronic HBV infection and upper aerodigestive tract, lung, kidney, skin, and thyroid gland cancers; lymphoma; and leukemia [16]. However, a case-control study in Shanghai, China, demonstrated that patients with HBV had no risk of cancers of the gallbladder, ampulla of Vater, and bile duct [17]. Overall, data on the association between $\mathrm{CHI}$ and extrahepatic cancer development in countries with endemic HBV and $\mathrm{HCV}$ infection are lacking. Previous studies have been conducted in countries with low prevalence and endemicity; hence, drawing a statistically supported conclusion from their results is difficult $[15,17,18]$. Moreover, these studies have focused on the association of HBV or HCV with primary liver cancer; comprehensive data on extrahepatic cancers among patients with $\mathrm{CHI}$ are lacking.

This longitudinal cohort study comprehensively investigated the association of $\mathrm{CHI}$ with extrahepatic cancer development in Taiwan, using a nationwide populationbased data set. HBV infection is endemic and HCV infection is highly prevalent in Taiwan [19]. In addition, cancer is highly prevalent in Taiwan, making the country an excellent setting for studying the association of $\mathrm{CHI}$ with cancer.

\section{Methods}

\section{Data sources}

In this study, the Longitudinal Health Insurance Database 2000 (LHID2000) of the National Health Insurance (NHI) program, which was launched in March 1995 to provide affordable healthcare services to all residents of Taiwan, was used. The program covered $93 \%$ of the population in 1997, and the coverage rate increased to approximately $99.9 \%$ by the end of 2014. The National Health Insurance Research Database (NHIRD) is a nationwide database extracted from the claims data of the NHI program for research purposes. This database contains information on inpatient and outpatient medical claims, including prescription and diagnosis records.

The LHID2000, which is a data set of the NHIRD, contains the claims data of one million beneficiaries randomly selected from all of the residents enrolled in the NHI program in 2000. No significant differences have been observed in age, sex, or healthcare costs between the entire population of this data set and all beneficiaries of the NHI program. Approval to use all the claims data and updated registries in the LHID2000 from 2000 to 2011 was received, and the International Classification of Diseases, Ninth Revision, Clinical Modification (ICD-9-CM) was used to identify disease diagnoses in the NHIRD. All data were anonymized upon inclusion in the NHIRD. Notably, this study was exempted from full review by the Institutional Review Board at China Medical University and the Hospital Research Ethics Committee (IRB permit number: CMU-REC-101-012).

\section{Study sample}

In this study, the association of $\mathrm{CHI}$ with hepatic and extrahepatic cancer development among an adult population ( $\geq 18$ years old) was investigated. The etiologies of other types of chronic hepatitis, such as autoimmune, chemical, and alcohol-related hepatitis, and nonalcoholic fatty liver disease, were excluded. Additionally, the presence of HBsAg was used as the major serum marker for cases of $\mathrm{HBV}$ infection recorded in the database. Patients with a history of human immunodeficiency virus (HIV) were excluded to minimize the inclusion of patients with HBV/HIV coinfection with occult HBV infection (i.e., HBsAg-negative patients with persistent HBV infection) [20]. Therefore, patients with a history of HIV (ICD-9-CM codes 042, 043, 044, V08, and 795.8) and chronic hepatitis (ICD-9-CM codes 571.4, 571.8, 571.9, and 573.3) without mention of HBV (ICD-9-CM codes $070.2,070.3$, and V02.61) or HCV (ICD-9-CM codes $070.41,070.44,070.51,070.54$, and V02.62) infection were excluded. The index date for patients with chronic $\mathrm{HBV}$ or $\mathrm{HCV}$ infection was the first date on which chronic HBV or HCV infection was detected. Patients with a diagnosis of cancer (ICD-9-CM codes 140-208) before the index date were also excluded. After applying the exclusion criteria, 15,888 patients with $\mathrm{HBV}$ infection (including 3,519 coinfected with $\mathrm{HCV}$ ) and 8,830 with $\mathrm{HCV}$ infection (including 3,519 coinfected with HBV), who were identified during 20002005, were enrolled in this study as the CHI cohort and followed up until cancer diagnosis or the end of 2011, whichever occurred first. In total, 939,971 insurants without hepatitis and with information on age and sex were identified; after excluding those aged $<18$ years who had had HIV or cancer before the index date, 63,552 and 35,320 control participants were identified and included in the non-HBV cohort and non-HCV cohort, respectively. The nonhepatitis cohorts were frequency matched to the CHI cohort at a ratio of $4: 1$ by age, sex, and index date and year (Fig. 1).

Patients newly diagnosed with head and neck cancer (ICD-9-CM codes 140 and 149), esophageal cancer (ICD9-CM code 150), stomach cancer (ICD-9-CM code 151), colorectal cancer (ICD-9-CM codes 153 and 154), liver cancer (ICD-9-CM code 155), gallbladder and extrahepatic bile duct cancer (ICD-9-CM code 156), pancreatic cancer (ICD-9-CM code 157), lung cancer (ICD-9-CM code 162), melanoma (ICD-9-CM code 172), skin cancer (ICD-9-CM code 173), breast cancer (ICD-9-CM codes 174 and 175), uterine and corpus cancer (ICD-9-CM codes 179 and 182), cervical cancer (ICD-9-CM code 180 ), ovarian cancer (ICD-9-CM code 183), prostate cancer (ICD-9-CM code 185), bladder cancer (ICD-9-CM code 188), kidney cancer (ICD-9-CM code 189), brain cancer (ICD-9-CM code 191), thyroid cancer (ICD-9-CM 


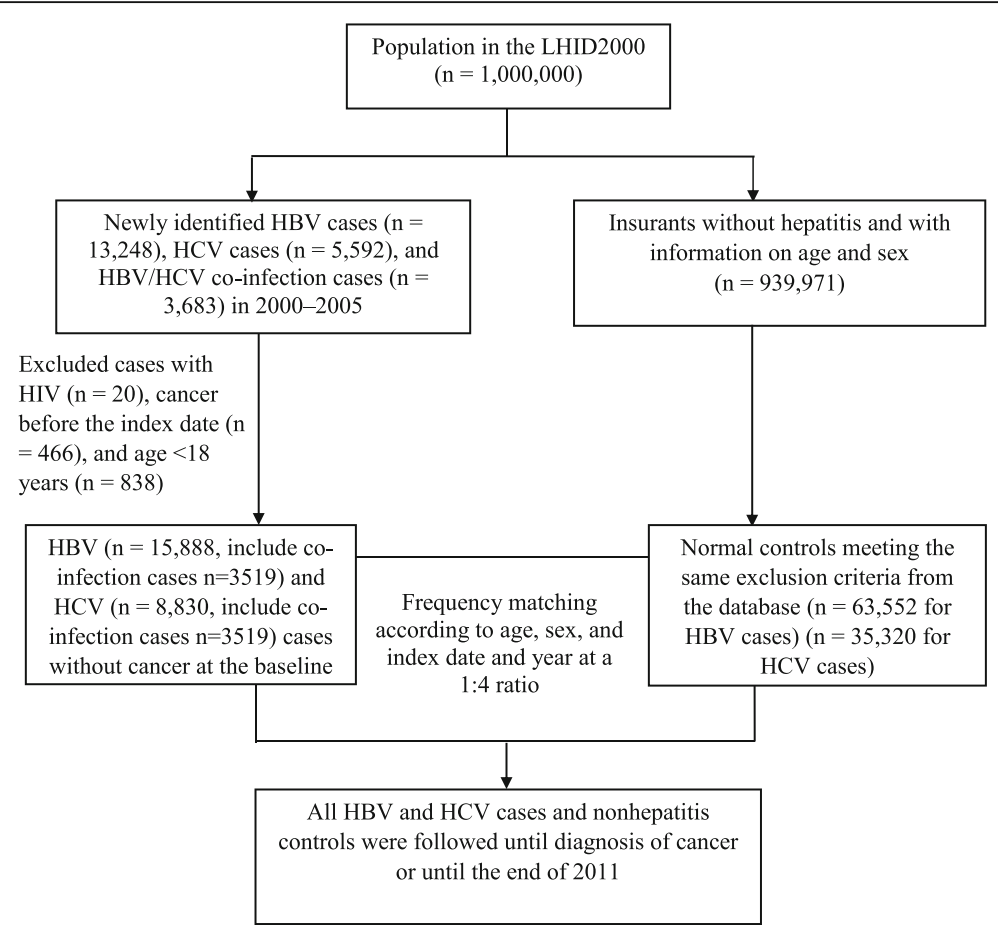

Fig. 1 Flowchart of patient recruitment

code 193), non-Hodgkin's lymphoma (ICD-9-CM code 202), myeloma (ICD-9-CM code 203), and leukemia (ICD-9-CM codes 204 and 208) during 2000-2011 were identified from the Registry of Catastrophic Illness Patients. Insurance coverage for catastrophic illnesses is an extension of the NHI program that protects people with serious disease against a devastating financial burden and subsequent impoverishment.

\section{Statistical analyses}

Pearson's chi-square test was used to compare the distributions of sociodemographic factors and various comorbidities, such as diabetes mellitus, hypertension, and hyperlipidemia, between the CHI cohort and the nonhepatitis cohorts, and the Student $t$-test was used to compare the number of outpatient visits between the CHI cohort and the nonhepatitis cohorts. Urbanization was categorized into four levels, with level 1 referring to the most urbanized communities and level 4 to the least urbanized communities. The geographical regions where the patients resided were divided into Northern Taiwan, Central Taiwan, Southern Taiwan, Eastern Taiwan, and the outlying islands. Additionally, the patients' monthly incomes were categorized into four groups: NT\$0, NT\$1NT\$15,840, NT\$15,841-NT\$25,000, and > NT\$25,000.

The cancer incidence rates were evaluated from the initial follow-up to the end of 2011. The follow-up period (years) was defined as the duration from chronic viral hepatitis identification to cancer diagnoses or censoring for death, emigration, or withdrawal from the NHI program, whichever occurred first. Poisson regression was used to calculate the incidence rate ratios with $95 \%$ confidence intervals (CIs) for comparison of our $\mathrm{HBV}$ or $\mathrm{HCV}$ cohorts with the adult population in the LHID2000. Finally, a Cox proportional hazard model was used to calculate hazard ratios (HRs) and $95 \%$ CIs for determining the association between $\mathrm{CHI}$ and cancer development. HRs were adjusted for sex, age, geographical region, occupation, level of urbanization, monthly income, the presence of comorbidities, and number of outpatient visits. A p value $<0.05$ was considered statistically significant. All statistical analyses were performed using SAS (Version 9.4 for Windows; SAS Institute, Inc., Cary, NC, USA).

\section{Results}

In our study, the patients with $\mathrm{HBV}$ infection were more likely to be laborers, reside in Central and Southern Taiwan, and have a higher monthly income, compared with the patients without HBV infection (Table 1). Moreover, these patients were more likely to have diabetes mellitus, hypertension, and hyperlipidemia. Similarly, the patients with $\mathrm{HCV}$ infection were more likely to be laborers, reside in less urbanized areas in Southern Taiwan, and have comorbidities, compared with the patients without HCV infection.

Table 2 presents the incidence densities of cancers among the patients with $\mathrm{CHI}$. The Poisson regression model revealed that, compared with the adult population 
Table 1 Baseline characteristics and comorbid conditions in hepatitis cohorts identified in 2000-2005

\begin{tabular}{|c|c|c|c|c|c|c|c|c|c|c|c|c|c|c|}
\hline \multirow[b]{2}{*}{ Variable } & \multicolumn{7}{|l|}{ HBV } & \multicolumn{7}{|l|}{$\mathrm{HCV}$} \\
\hline & $\begin{array}{l}\text { No } \\
n(\%)\end{array}$ & & $\begin{array}{l}\text { Yes } \\
n(\%)\end{array}$ & & $x^{2}$ & df & $p$ value & $\begin{array}{l}\text { No } \\
n(\%)\end{array}$ & & $\begin{array}{l}\text { Yes } \\
n(\%)\end{array}$ & & $x^{2}$ & df & $P$ value \\
\hline Women & 26832 & $(42.2)$ & 6708 & $(42.2)$ & & & & 16864 & $(47.7)$ & 4216 & $(47.7)$ & & & \\
\hline Men & 36720 & $(57.8)$ & 9180 & (57.8) & & & & 18456 & $(52.3)$ & 4614 & $(52.3)$ & & & \\
\hline Age, years & & & & & 0.00 & 3 & 1.000 & & & & & 0.00 & 3 & 1.000 \\
\hline$<50$ & 45564 & $(71.7)$ & 11391 & $(71.7)$ & & & & 17116 & $(48.5)$ & 4279 & $(48.5)$ & & & \\
\hline $50-59$ & 9308 & $(14.6)$ & 2327 & $(14.6)$ & & & & 7284 & $(20.6)$ & 1821 & (20.6) & & & \\
\hline $60-69$ & 5896 & $(9.3)$ & 1474 & (9.3) & & & & 6784 & $(19.2)$ & 1696 & $(19.2)$ & & & \\
\hline$\geq 70$ & 2784 & $(4.4)$ & 696 & $(4.4)$ & & & & 4136 & $(11.7)$ & 1034 & $(11.7)$ & & & \\
\hline Geographical region & & & & & 212.15 & 3 & $<0.0001$ & & & & & 967.58 & 3 & $<0.0001$ \\
\hline Northern & 30226 & $(47.6)$ & 6594 & $(41.5)$ & & & & 16176 & $(45.8)$ & 2666 & $(30.2)$ & & & \\
\hline Central & 12404 & $(19.5)$ & 3401 & (21.4) & & & & 6962 & $(19.7)$ & 1882 & $(21.3)$ & & & \\
\hline Southern & 16079 & $(25.3)$ & 4698 & (29.6) & & & & 9110 & $(25.8)$ & 3597 & $(40.7)$ & & & \\
\hline Eastern and islands & 4843 & (7.6) & 1195 & $(7.5)$ & & & & 3072 & $(8.7)$ & 685 & (7.8) & & & \\
\hline Occupation & & & & & 118.28 & 4 & $<0.0001$ & & & & & 277.96 & 4 & $<0.0001$ \\
\hline Public & 5956 & (9.4) & 1829 & (11.5) & & & & 3519 & $(10.0)$ & 758 & (8.6) & & & \\
\hline Labor & 19351 & $(30.4)$ & 5159 & (32.5) & & & & 12796 & $(36.2)$ & 4010 & $(45.4)$ & & & \\
\hline Business & 30002 & $(47.2)$ & 7070 & $(44.5)$ & & & & 14066 & (39.8) & 2879 & (32.6) & & & \\
\hline Low income & 256 & $(0.4)$ & 65 & $(0.4)$ & & & & 183 & $(0.5)$ & 68 & $(0.8)$ & & & \\
\hline Retired & 7987 & $(12.6)$ & 1765 & (11.1) & & & & 4756 & $(13.5)$ & 1115 & $(12.6)$ & & & \\
\hline Urbanization level & & & & & 94.02 & 3 & $<0.0001$ & & & & & 428.06 & 3 & $<0.0001$ \\
\hline 1 (highest) & 19662 & $(30.9)$ & 4431 & (27.9) & & & & 10499 & $(29.7)$ & 1873 & $(21.2)$ & & & \\
\hline 2 & 18754 & $(29.5)$ & 4713 & (29.7) & & & & 10162 & $(28.8)$ & 2531 & $(28.7)$ & & & \\
\hline 3 & 11896 & $(18.7)$ & 2952 & (18.6) & & & & 6314 & $(17.9)$ & 1513 & $(17.1)$ & & & \\
\hline 4 (lowest) & 13232 & (20.8) & 3791 & (23.9) & & & & 8340 & $(23.6)$ & 2912 & (33.0) & & & \\
\hline Monthly income, NT\$ & & & & & 79.93 & 3 & $<0.0001$ & & & & & 127.57 & 3 & $<0.0001$ \\
\hline 0 & 13698 & $(21.6)$ & 3282 & (20.7) & & & & 8233 & $(23.3)$ & 1869 & $(21.2)$ & & & \\
\hline $1-15,840$ & 7712 & $(12.1)$ & 1633 & $(10.3)$ & & & & 4180 & $(11.8)$ & 990 & $(11.2)$ & & & \\
\hline $15,841-25,000$ & 27135 & $(42.7)$ & 6795 & $(42.8)$ & & & & 15717 & $(44.5)$ & 4495 & $(50.9)$ & & & \\
\hline$>25,000$ & 15007 & $(23.6)$ & 4178 & (26.3) & & & & 7190 & $(20.4)$ & 1476 & $(16.7)$ & & & \\
\hline Diabetes mellitus & & & & & 232.42 & 1 & $<0.0001$ & & & & & 280.01 & 1 & $<0.0001$ \\
\hline No & 59599 & $(93.8)$ & 14355 & $(90.4)$ & & & & 31531 & $(89.3)$ & 7311 & $(82.8)$ & & & \\
\hline Yes & 3953 & $(6.2)$ & 1533 & (9.6) & & & & 3789 & $(10.7)$ & 1519 & $(17.2)$ & & & \\
\hline Hypertension & & & & & 88.26 & 1 & $<0.0001$ & & & & & 122.42 & 1 & $<0.0001$ \\
\hline No & 54129 & $(85.2)$ & 13054 & $(82.2)$ & & & & 26215 & $(74.2)$ & 6038 & $(68.4)$ & & & \\
\hline Yes & 9423 & $(14.8)$ & 2834 & $(17.8)$ & & & & 9105 & $(25.8)$ & 2792 & (31.6) & & & \\
\hline Hyperlipidemia & & & & & 347.37 & 1 & $<0.0001$ & & & & & 121.67 & 1 & $<0.0001$ \\
\hline No & 57936 & $(91.2)$ & 13702 & $(86.2)$ & & & & 30422 & $(86.1)$ & 7194 & $(81.5)$ & & & \\
\hline Yes & 5616 & (8.8) & 2186 & (13.8) & & & & 4898 & $(13.9)$ & 1636 & $(18.5)$ & & & \\
\hline Outpatient visits, mean (SD) & 12 & (13) & 16 & (15) & & & $<0.0001^{\mathrm{a}}$ & 14 & $(15)$ & 21 & (18) & & & $<0.0001^{a}$ \\
\hline
\end{tabular}


Table 2 Incidence densities of cancers in patients diagnosed with chronic HBV and HCV infection

\begin{tabular}{|c|c|c|c|c|c|c|c|c|c|c|}
\hline \multirow[b]{2}{*}{ Cancer type } & \multicolumn{2}{|c|}{ All LHID2000 } & \multirow[b]{2}{*}{ Events } & \multicolumn{2}{|l|}{ HBV } & \multirow[b]{2}{*}{$(95 \%$ Cl) } & \multirow[b]{2}{*}{ Events } & \multicolumn{2}{|l|}{$\mathrm{HCV}$} & \multirow[b]{2}{*}{$(95 \% \mathrm{Cl})$} \\
\hline & Events & $\overline{\text { Rate }^{a}}$ & & Rate $^{a}$ & $\mathrm{IRR}^{\mathrm{b}}$ & & & $\overline{\text { Rate }^{a}}$ & $\mathrm{IRR}^{\mathrm{b}}$ & \\
\hline Overall & 40213 & 36.59 & 1330 & 94.01 & 2.95 & $(2.80-3.12)^{*}$ & 1232 & 163.00 & 3.33 & $(3.15-3.53)$ \\
\hline Brain cancer & 509 & 0.46 & 7 & 0.49 & 1.13 & $(0.53-2.38)$ & 7 & 0.93 & 1.61 & $(0.76-3.39)$ \\
\hline Head and neck cancer & 4079 & 3.71 & 66 & 4.67 & 1.23 & $(0.97-1.57)$ & 48 & 6.35 & 1.43 & $(1.08-1.90)^{*}$ \\
\hline Thyroid gland cancer & 1138 & 1.04 & 24 & 1.70 & 1.78 & $(1.19-2.67)^{*}$ & 13 & 1.72 & 1.53 & $(0.89-2.65)$ \\
\hline Esophageal cancer & 850 & 0.77 & 15 & 1.06 & 1.45 & $(0.87-2.42)$ & 11 & 1.46 & 1.48 & $(0.82-2.68)$ \\
\hline Stomach cancer & 2247 & 2.04 & 32 & 2.26 & 1.42 & $(1.00-2.01)^{*}$ & 37 & 4.90 & 1.78 & $(1.28-2.46)^{*}$ \\
\hline Colorectal cancer & 5890 & 5.36 & 104 & 7.35 & 1.69 & $(1.39-2.05)^{*}$ & 83 & 10.98 & 1.51 & $(1.21-1.87)^{*}$ \\
\hline Liver cancer & 5293 & 4.82 & 770 & 54.43 & 12.89 & $(11.9-13.9)^{*}$ & 782 & 103.46 & 16.26 & $(15.1-17.5)^{*}$ \\
\hline Gallbladder and extrahepatic bile duct cancer & 501 & 0.46 & 14 & 0.99 & 2.80 & $(1.64-4.76)^{*}$ & 18 & 2.38 & 3.81 & $(2.38-6.10)^{*}$ \\
\hline Pancreatic cancer & 684 & 0.62 & 19 & 1.34 & 2.73 & $(1.73-4.31)^{*}$ & 17 & 2.25 & 2.66 & $(1.65-4.31)^{*}$ \\
\hline Lung cancer & 4656 & 4.24 & 74 & 5.23 & 1.57 & $(1.25-1.97)^{*}$ & 69 & 9.13 & 1.61 & $1.27-2.04)^{*}$ \\
\hline Kidney cancer & 1065 & 0.97 & 28 & 1.98 & 2.47 & $(1.70-3.60)^{*}$ & 17 & 2.25 & 1.71 & $(1.06-2.76)^{*}$ \\
\hline Bladder cancer & 1324 & 1.20 & 24 & 1.70 & 1.83 & $(1.22-2.74)^{*}$ & 24 & 3.18 & 1.98 & $(1.33-2.97)^{*}$ \\
\hline Uterine and corpus cancer ${ }^{c}$ & 600 & 1.10 & 14 & 2.29 & 2.12 & $(1.25-3.61)^{*}$ & 11 & 2.98 & 2.17 & $(1.19-3.93)^{*}$ \\
\hline Cervical cancer $^{c}$ & 1877 & 3.43 & 10 & 1.64 & 0.51 & $(0.27-0.95)$ & 11 & 2.98 & 0.62 & $(0.34-1.12)$ \\
\hline Ovarian cancer $^{c}$ & 592 & 1.08 & 15 & 2.45 & 2.29 & $(1.37-3.82)^{*}$ & 8 & 2.16 & 1.67 & $(0.83-3.35)$ \\
\hline Prostate cancer $^{\mathrm{d}}$ & 1820 & 3.30 & 30 & 3.73 & 1.83 & $(1.28-2.63)^{*}$ & 24 & 6.21 & 1.55 & $(1.04-2.32)^{*}$ \\
\hline Breast cancer ${ }^{c}$ & 4695 & 8.58 & 74 & 12.10 & 1.43 & $(1.14-1.80)^{*}$ & 46 & 12.45 & 1.17 & $(0.87-1.56)$ \\
\hline Melanoma & 136 & 0.12 & 0 & - & - & - & 2 & 0.26 & 1.58 & $(0.39-6.37)$ \\
\hline Skin cancer & 619 & 0.56 & 15 & 1.06 & 2.44 & $(1.46-4.08)^{*}$ & 11 & 1.46 & 1.90 & $(1.04-3.44)^{*}$ \\
\hline Non-Hodgkin's lymphoma & 738 & 0.67 & 22 & 1.56 & 2.58 & $(1.69-3.94)^{*}$ & 20 & 2.65 & 3.01 & $(1.93-4.69)^{*}$ \\
\hline Myeloma & 218 & 0.20 & 2 & 0.14 & 0.87 & $(0.22-3.49)$ & 3 & 0.40 & 1.47 & $(0.47-4.60)$ \\
\hline Leukemia & 682 & 0.62 & 15 & 1.06 & 1.82 & $(1.09-3.04)^{*}$ & 7 & 0.93 & 1.19 & $(0.56-2.50)$ \\
\hline
\end{tabular}

IRR incidence rate ratio

${ }^{a}$ Per 10,000 person-years

${ }^{\mathrm{b}}$ Adjusted for sex and age

'Women only

${ }^{\mathrm{d}}$ Men only

${ }^{*} p<0.05$

in the LHID2000, the patients with HBV or HCV infection exhibited an increased risk of liver cancer (HR: 12.89, 95 \% CI: 11.9-13.9 or HR: 16.26, 95 \% CI: 15.1-17.5, respectively). In addition, HBV infection was associated with an increased risk of developing thyroid gland cancer, stomach cancer, colorectal cancer, gallbladder and extrahepatic bile duct cancer, pancreatic cancer, lung cancer, kidney cancer, bladder cancer, uterine and corpus cancer, ovarian cancer, prostate cancer, breast cancer, skin cancer, non-Hodgkin's lymphoma, and leukemia. Similarly, HCV infection was associated with an increased risk of developing head and neck cancer, stomach cancer, colon and rectum cancer, gallbladder and bile duct cancer, pancreatic cancer, lung cancer, kidney cancer, bladder cancer, uterine cancer, prostate cancer, skin cancer, and non-Hodgkin's lymphoma.

Compared with their corresponding nonhepatitis cohorts, the overall adjusted HRs for the risk of various cancers were 2.67 (95 \% CI: 2.49-2.86) and 2.83 (95\% CI: 2.63-3.05) for the HBV and HCV cohorts, respectively (Table 3 ). Specifically, the patients with HBV infection exhibited an increased risk of colorectal cancer (HR: 1.36, 95 \% CI: 1.09-1.70), liver cancer (HR: 21.47, 95 \% CI: 18.0-25.6), gallbladder and extrahepatic bile cancer (HR: 2.05, 95 \% CI: 1.07-3.91), pancreatic cancer (HR: 2.61, 95 \% CI: 1.47-4.61), kidney cancer (HR: 1.72, 95 \% CI: 1.10-2.68), ovarian cancer (HR: $2.31,95$ \% CI: 1.21-4.39), and non-Hodgkin's lymphoma (HR: 2.10, 95 \% CI: 1.25-3.52). Further analysis revealed that HCV was also a significant risk factor for liver cancer (HR: 25.10, 95 \% CI: 20.9-30.2), gallbladder and extrahepatic bile duct cancer (HR: 2.60, 95 \% CI: 1.42-4.73), ovarian cancer (HR: 5.15, 95 \% CI: 1.98-13.4), and nonHodgkin's lymphoma (HR: 2.30, 95 \% CI: 1.34-3.96).

Furthermore, we analyzed our data after excluding those with $\mathrm{HBV} / \mathrm{HCV}$ coinfection. The patients with 
Table 3 Hazard ratios for developing cancer in patients with $\mathrm{CHI}$

\begin{tabular}{|c|c|c|c|c|c|c|c|c|}
\hline \multirow[b]{3}{*}{ Cancer type } & \multicolumn{4}{|l|}{$\underline{\mathrm{HBV}}$} & \multicolumn{4}{|l|}{$\underline{\mathrm{HCV}}$} \\
\hline & \multirow{2}{*}{$\begin{array}{l}\text { No } \\
\text { Cases }\end{array}$} & \multirow{2}{*}{$\begin{array}{l}\text { Yes } \\
\text { Cases }\end{array}$} & \multirow[b]{2}{*}{$\mathrm{HR}$} & \multirow[b]{2}{*}{$(95 \% \mathrm{Cl})^{\mathrm{a}}$} & \multirow{2}{*}{$\begin{array}{l}\text { No } \\
\text { Cases }\end{array}$} & \multirow{2}{*}{$\begin{array}{l}\text { Yes } \\
\text { Cases }\end{array}$} & \multirow[b]{2}{*}{$\mathrm{HR}$} & \multirow[b]{2}{*}{$(95 \% \mathrm{Cl})^{\mathrm{a}}$} \\
\hline & & & & & & & & \\
\hline Overall & 2,139 & 1,330 & 2.67 & $(2.49-2.86)^{\ddagger}$ & 1,893 & 1,232 & 2.83 & $(2.63-3.05)^{\ddagger}$ \\
\hline Brain cancer & 30 & 7 & 1.04 & $(0.45-2.39)$ & 18 & 7 & 1.67 & $(0.68-4.15)$ \\
\hline Head and neck cancer & 288 & 66 & 0.96 & $(0.73-1.26)$ & 171 & 48 & 1.13 & $(0.81-1.57)$ \\
\hline Thyroid gland cancer & 61 & 24 & 1.50 & $(0.93-2.42)$ & 44 & 13 & 1.18 & $(0.62-2.24)$ \\
\hline Esophageal cancer & 54 & 15 & 1.42 & $(0.79-2.54)$ & 46 & 11 & 1.24 & $(0.63-2.43)$ \\
\hline Stomach cancer & 132 & 32 & 1.11 & $(0.75-1.65)$ & 122 & 37 & 1.41 & $(0.96-2.06)$ \\
\hline Colorectal cancer & 341 & 104 & 1.36 & $(1.09-1.70)^{\dagger}$ & 327 & 83 & 1.07 & $(0.84-1.38)$ \\
\hline Liver cancer & 155 & 770 & 1.47 & $(18.0-25.6)^{\ddagger}$ & 137 & 782 & 25.10 & $(20.9-30.2)^{\ddagger}$ \\
\hline Gallbladder and extrahepatic bile duct cancer & 30 & 14 & 2.05 & $(1.07-3.91)^{*}$ & 32 & 18 & 2.60 & $(1.42-4.73)^{\dagger}$ \\
\hline Pancreatic cancer & 35 & 19 & 2.61 & $(1.47-4.61)^{\dagger}$ & 55 & 17 & 1.51 & $(0.86-2.65)$ \\
\hline Lung cancer & 292 & 74 & 1.11 & $(0.86-1.44)$ & 318 & 69 & 0.93 & $(0.71-1.21)$ \\
\hline Kidney cancer & 69 & 28 & 1.72 & $(1.10-2.68)^{*}$ & 68 & 17 & 0.99 & $(0.57-1.71)$ \\
\hline Bladder cancer & 80 & 24 & 1.24 & $(0.78-1.97)$ & 87 & 24 & 1.20 & $(0.75-1.90)$ \\
\hline Uterine and corpus cancer ${ }^{\mathrm{b}}$ & 43 & 14 & 1.28 & $(0.69-2.37)$ & 26 & 11 & 1.82 & $(0.87-3.78)$ \\
\hline Cervical cancer $^{\mathrm{b}}$ & 62 & 10 & 0.74 & $(0.38-1.46)$ & 62 & 11 & 0.81 & $(0.42-1.56)$ \\
\hline Ovarian cancer ${ }^{\mathrm{b}}$ & 27 & 15 & 2.31 & $(1.21-4.39)^{*}$ & 10 & 8 & 5.15 & $(1.98-13.4)^{\dagger}$ \\
\hline Prostate cancer $^{c}$ & 134 & 30 & 0.95 & $(0.64-1.42)$ & 136 & 24 & 0.81 & $(0.52-1.27)$ \\
\hline Breast cancer ${ }^{b}$ & 256 & 74 & 1.25 & $(0.96-1.62)$ & 197 & 46 & 1.08 & $(0.78-1.50)$ \\
\hline Melanoma & 14 & 0 & - & - & 12 & 2 & 0.78 & $(0.17-3.60)$ \\
\hline Skin cancer & 37 & 15 & 1.46 & $(0.79-2.69)$ & 37 & 11 & 1.09 & $(0.54-2.17)$ \\
\hline Non-Hodgkin's lymphoma & 45 & 22 & 2.10 & $(1.25-3.52)^{\dagger}$ & 45 & 20 & 2.30 & $(1.34-3.96)^{\dagger}$ \\
\hline Myeloma & 12 & 2 & 0.93 & $(0.20-4.25)$ & 13 & 3 & 1.15 & $(0.32-4.20)$ \\
\hline Leukemia & 43 & 15 & 1.44 & $(0.79-2.61)$ & 25 & 7 & 1.21 & $(0.51-2.87)$ \\
\hline
\end{tabular}

${ }^{*} p<0.05,{ }^{+} p<0.001,{ }^{\ddagger} p<0.0001$

${ }^{a}$ Adjusted for sex, age, geographical region, occupation, level of urbanization, monthly income, the presence of comorbidities, and number of outpatient visits bomen only

cMen only

only HBV infection exhibited an increased risk of colorectal cancer (HR: 1.51, 95 \% CI: 1.15-1.98), liver cancer (HR: 18.9, 95 \% CI: 15.2-23.6), kidney cancer (HR: 1.81, 95 \% CI: 1.10-3.01), and non-Hodgkin's lymphoma (HR: 2.22, $95 \%$ CI: 1.18-4.18), whereas the patients with $\mathrm{HCV}$ exhibited an increased risk of liver cancer (HR: 23.28, 95 \% CI: 18.4-29.5), gallbladder and extrahepatic bile duct cancer (HR: 2.53, $95 \%$ CI: $1.17-5.48$ ), and non-Hodgkin's lymphoma (HR: 2.66, 95 \% CI: 1.34-5.27) (Table 4).

\section{Discussion}

The present population-based study revealed that $\mathrm{CHI}$ is associated with an increased risk of extrahepatic cancer in Taiwan. Approximately $15.4 \%$ of the global cancer burden can be attributed to five infectious agents, namely Epstein-Barr virus, human papillomavirus, HBV, $\mathrm{HCV}$, and Helicobacter pylori [21]. HBV, an enveloped DNA virus from the hepadnavirus family, has a high affinity for hepatocytes. In Asia, where HBV infection is highly endemic, vertical transmission is the main route of $\mathrm{HBV}$ exposure. By contrast, $\mathrm{HCV}$ is an RNA virus from the flavivirus family that is commonly transmitted horizontally through contaminated blood, blood products, and intravenous drug use.

The association between $\mathrm{CHI}$ and primary liver cancer has been extensively documented [7, 22-24], and in this study, the patients with $\mathrm{CHI}$ exhibited an increased risk of liver cancer. However, the mechanism by which hepatitis viruses cause liver cancer remains unclear. It has been suggested that hepatitis viruses cause genomic instability through integration into human chromosomes, which causes the chromosomal rearrangement of cellular genes and increases the likelihood of hepatocarcinogenesis [25]. Furthermore, patients with $\mathrm{CHI}$ are at an increased risk of non-Hodgkin's lymphoma [12-15]. In this study, the patients with $\mathrm{CHI}$ exhibited a higher risk of non-Hodgkin's lymphoma than the nonhepatitis cohorts. 
Table 4 Hazard ratios for developing cancer in patients with $\mathrm{CHI}$ after excluding HBV/HCV coinfected patients

\begin{tabular}{|c|c|c|c|c|c|c|c|c|}
\hline \multirow[b]{3}{*}{ Cancer type } & \multicolumn{4}{|l|}{ HBV } & \multicolumn{4}{|l|}{$\mathrm{HCV}$} \\
\hline & \multirow{2}{*}{$\begin{array}{l}\text { No } \\
\text { Cases }\end{array}$} & \multirow{2}{*}{$\begin{array}{l}\text { Yes } \\
\text { Cases }\end{array}$} & \multirow[b]{2}{*}{$\mathrm{HR}$} & \multirow[b]{2}{*}{$(95 \% \mathrm{Cl})^{a}$} & \multirow{2}{*}{$\begin{array}{l}\text { No } \\
\text { Cases }\end{array}$} & \multirow{2}{*}{$\begin{array}{l}\text { Yes } \\
\text { Cases }\end{array}$} & \multirow[b]{2}{*}{$H R$} & \multirow[b]{2}{*}{$(95 \% \mathrm{Cl})^{a}$} \\
\hline & & & & & & & & \\
\hline Overall & 1,517 & 829 & 2.34 & $(2.15-2.55)^{\ddagger}$ & 1,174 & 731 & 2.71 & $(2.47-2.99)^{\ddagger}$ \\
\hline Brain cancer & 21 & 4 & 0.85 & $(0.29-2.51)$ & 13 & 4 & 1.51 & $(0.48-4.77)$ \\
\hline Head and neck cancer & 233 & 47 & 0.84 & $(0.61-1.16)$ & 107 & 29 & 1.06 & $(0.70-1.62)$ \\
\hline Thyroid gland cancer & 53 & 20 & 1.41 & $(0.84-2.38)$ & 23 & 9 & 1.62 & $(0.73-3.61)$ \\
\hline Esophageal cancer & 42 & 13 & 1.47 & $(0.78-2.76)$ & 22 & 9 & 2.08 & $(0.93-4.65)$ \\
\hline Stomach cancer & 78 & 18 & 1.01 & $(0.60-1.69)$ & 76 & 23 & 1.38 & $(0.85-2.24)$ \\
\hline Colorectal cancer & 212 & 72 & 1.51 & $(1.15-1.98)^{\dagger}$ & 204 & 51 & 1.09 & $(0.79-1.49)$ \\
\hline Liver cancer & 102 & 439 & 18.9 & $(15.2-23.6)^{\ddagger}$ & 86 & 451 & 23.28 & $(18.4-29.5)^{\ddagger}$ \\
\hline Gallbladder and extrahepatic bile duct cancer & 20 & 7 & 1.56 & $(0.65-3.73)$ & 19 & 11 & 2.53 & $(1.17-5.48)^{*}$ \\
\hline Pancreatic cancer & 33 & 13 & 1.69 & $(0.88-3.23)$ & 32 & 11 & 1.68 & $(0.82-3.41)$ \\
\hline Lung cancer & 198 & 48 & 1.07 & $(0.78-1.47)$ & 212 & 43 & 0.87 & $(0.62-1.21)$ \\
\hline Kidney cancer & 52 & 22 & 1.81 & $(1.10-3.01)^{*}$ & 47 & 11 & 0.98 & $(0.49-1.93)$ \\
\hline Bladder cancer & 67 & 13 & 0.87 & $(0.48-1.58)$ & 65 & 13 & 0.91 & $(0.50-1.68)$ \\
\hline Uterine and corpus cancer ${ }^{\mathrm{b}}$ & 33 & 11 & 1.26 & $(0.63-2.50)$ & 25 & 8 & 1.61 & $(0.71-3.67)$ \\
\hline Cervical cancer $^{\mathrm{b}}$ & 42 & 10 & 1.10 & $(0.55-2.21)$ & 32 & 11 & 1.52 & $(0.75-3.08)$ \\
\hline Ovarian cancer ${ }^{\mathrm{b}}$ & 23 & 8 & 1.35 & $(0.60-3.07)$ & 10 & 1 & 0.58 & $(0.07-4.62)$ \\
\hline Prostate cancer ${ }^{c}$ & 96 & 22 & 0.93 & $(0.58-1.49)$ & 82 & 16 & 0.84 & $(0.48-1.46)$ \\
\hline Breast cancer $^{\mathrm{b}}$ & 192 & 53 & 1.15 & $(0.84-1.56)$ & 92 & 25 & 1.24 & $(0.78-1.95)$ \\
\hline Melanoma & 11 & 0 & - & - & 7 & 2 & 1.48 & $(0.29-7.56)$ \\
\hline Skin cancer & 27 & 13 & 1.90 & $(0.97-3.73)$ & 24 & 9 & 1.35 & $(0.61-2.99)$ \\
\hline Non-Hodgkin's lymphoma & 29 & 15 & 2.22 & $(1.18-4.18)^{*}$ & 27 & 13 & 2.66 & $(1.34-5.27)^{\dagger}$ \\
\hline Myeloma & 9 & 2 & 1.04 & $(0.22-4.90)$ & 5 & 3 & 3.25 & $(0.73-14.5)$ \\
\hline Leukemia & 35 & 10 & 1.24 & $(0.61-2.52)$ & 22 & 2 & 0.35 & $(0.08-1.53)$ \\
\hline
\end{tabular}

${ }^{*} p<0.05,{ }^{+} p<0.001,{ }^{\ddagger} p<0.0001$

${ }^{a}$ Adjusted for sex, age, geographical region, occupation, level of urbanization, monthly income, the presence of comorbidities, and number of outpatient visits bomen only

cMen only

This finding supports and extends previous reports of a significant association between CHI and non-Hodgkin's lymphoma. Moreover, the patients with $\mathrm{CHI}$ exhibited an increased risk of gallbladder and extrahepatic bile duct cancer, which confirms that patients with $\mathrm{CHI}$ are at an increased risk of cancer [11].

In addition to the well-established association between $\mathrm{CHI}$ and primary liver cancer, our results indicated that HBV infection is associated with an increased risk of pancreatic cancer. However, the association was nonsignificant after excluding the patients with $\mathrm{HBV} / \mathrm{HCV}$ coinfection. This observation is attributed to the small sample size for pancreatic cancer; hence, statistical power was decreased. The pancreas serves as a potential reservoir of hepatitis viruses because of its close proximity to the liver, and the blood vessels and ducts it shares with the liver [26]. Thus, the increased risk of pancreatic cancer among the patients with HBV can be attributed to these two factors. Moreover, previous meta-analyses have reported an increased risk of pancreatic cancer among patients with HBV infection [27, 28], which corroborate our findings.

In the present study, other cancers such as kidney cancer, colorectal cancer, and ovarian cancer were also associated with $\mathrm{CHI}$. Sundquist et al. determined that there was an increased incidence of kidney cancer among patients with HBV, substantiating our results [16]. Nevertheless, the increased risk of kidney cancer observed here is a novel finding that requires further investigation. Only a few studies have investigated the association between $\mathrm{CHI}$ and colorectal cancer, and they have reported inconsistent findings $[18,29]$. Rustagi et al. demonstrated that $\mathrm{HCV}$ is an independent risk factor for colorectal adenoma, and reported that $\mathrm{HCV}$ is associated with a 2.04-fold higher risk of colorectal cancer [29]. By contrast, in this study, we only observed this association among the patients with HBV. In addition, previous studies have suggested that $\mathrm{X}$ protein from $\mathrm{HBV}$ can 
bind and interfere with the components of the DNA repair machinery and p53 tumor suppressor in response to DNA damage, thereby increasing the risk of colorectal cancer $[18,30]$. In addition, the HBV X protein has been reported to be highly expressed in the ovarian cancer cells of Chinese women, implying that it may be involved in the carcinogenesis of ovarian cancer [31]. However, the association between $\mathrm{CHI}$ and the aforementioned cancers was nonsignificant after excluding the patients with $\mathrm{HBV} / \mathrm{HCV}$ coinfection, which reduced the statistical power of the study.

By contrast, Mahale et al. and Lee et al. have argued that there is an increased risk of head and neck, prostate, and esophageal cancers among patients with $\mathrm{HCV}$ $[32,33]$. However, a nonsignificant association was observed between $\mathrm{CHI}$ and these cancers in this study, which is consistent with the findings of similar research in the United States $[34,35]$. This discrepancy can be attributed to the control group selected by Mahale et al., which included patients diagnosed with lung, esophageal, and urinary bladder cancers [32]. Moreover, the novel findings reported by Lee et al. can be attributed to the study's small sample size for esophageal and prostate cancers [33].

A major strength of the present study is that it analyzed a large cohort of patients identified from the NHIRD, covering nearly the entire population of Taiwan. Only patients diagnosed with HIV infection, alcohol-related hepatitis, and autoimmune hepatitis were excluded, to prevent the confounding effects of these diseases from skewing our results. However, this study has some limitations. First, the NHIRD does not contain detailed information about patients' smoking habits, alcohol consumption, family cancer history, body mass index, nutritional status, environmental exposure to chemicals, or history of substance use; therefore, we could not rule out the potential confounding effects of these factors. Another limitation is the small sample size for specific rare cancers, which reduced the statistical power of this study.

\section{Conclusions}

In addition to the well-established association between $\mathrm{CHI}$ and primary liver cancer, the present populationbased cohort study revealed that $\mathrm{CHI}$ is associated with an increased risk of extrahepatic cancers of the colon and rectum, gallbladder and extrahepatic bile ducts, pancreas, kidneys, and ovaries, as well as non-Hodgkin's lymphoma.

\footnotetext{
Abbreviations

CHI: Chronic hepatitis infection; Cl: Confidence interval; HBsAg: Hepatitis B surface antigen; HBV: Hepatitis B virus; HCV: Hepatitis C virus; HIV: Human immunodeficiency virus; HR: Hazard ratio; ICD-9-CM: International classification of diseases, ninth revision, clinical modification; LHID2000: Longitudinal health insurance database 2000; NHI: National health insurance; NHIRD: National Health insurance research database
}

\section{Acknowledgment}

We would like to thank Taiwan National Health Insurance Research Database for providing the data set used in this study.

\section{Funding}

This study was supported in part by grants from the Taiwan Ministry of Health and Welfare Clinical Trial and Research Center of Excellence (MOHW104-TDU-B-212-113002, MOHW105-TDU-B-212-133019), China Medical University Hospital, the Academia Sinica Taiwan Biobank Stroke Biosignature Project (BM104010092), the NRPB Stroke Clinical Trial Consortium (MOST 1032325-B-039-006), and Taipei Medical University (TMU-NTUST-103-11). The funders had no role in study design, data collection and analysis, decision to publish, or preparation of the manuscript.

\section{Availability of data and material}

All data are available from the NHIRD of Taiwan (http://nhird.nhri.org.tw/) Requests for data can be sent as a formal proposal to the NHIRD.

\section{Authors' contributions}

ABK, FHS, and CCY conceived and designed the experiments. ABK, FHS, WCW, FCS, SNC, and CCY performed the experiments. WCW, FCS, and SNC analyzed the data. WCW, FCS, SNC, and CCY contributed reagents, materials, and analysis tools. ABK and CCY wrote the paper. All authors directly participated in the planning, execution, or analysis of the study, and read and approved the final version submitted.

\section{Competing interests}

The authors declare that they have no competing interests.

\section{Consent for publication}

Not applicable.

\section{Ethics approval and consent to participate}

This study was exempted from a full review by the Institutional Review Board of China Medical University and the Hospital Research Ethics

Committee (IRB permit number: CMU-REC-101-012).

\section{Author details}

${ }^{1}$ School of Public Health, College of Public Health and Nutrition, Taipei Medical University, No. 250 Wu-Hsing Street, Taipei 11031, Taiwan. ${ }^{2}$ Department of Family Medicine, School of Medicine, College of Medicine, Taipei Medical University, No. 250 Wu-Hsing Street, Taipei 11031, Taiwan. ${ }^{3}$ Department of Family Medicine, Taipei Medical University Hospital, No. 252 Wu-Hsing Street, Taipei 11031, Taiwan. ${ }^{4}$ Master Program in Long-Term Care, College of Nursing, Taipei Medical University, No. 250 Wu-Hsing Street, Taipei 11031, Taiwan. ${ }^{5}$ School of Medicine, Flinders University, Bedford Park, Australia. ${ }^{6}$ The Ph.D. Program for Translational Medicine, College of Medical Science and Technology, Taipei Medical University, No. 250 Wu-Hsing Street, Taipei 11031, Taiwan. ${ }^{7}$ Management Office for Health Data, China Medical University Hospital, No. 2 Yude Road, Taichung 40402, Taiwan. ${ }^{8}$ Graduate Institute of Clinical Medical Science, School of Medicine, College of Medicine, China Medical University, No. 91 Hsueh-Shih Road, Taichung 40402, Taiwan. ${ }^{9}$ Department of Public Health, China Medical University, No. 91 Hsueh-Shih Road, Taichung 40402, Taiwan.

Received: 13 July 2016 Accepted: 31 October 2016

Published online: 08 November 2016

\section{References}

1. World Health Organization. Hepatitis B vaccines. Wkly Epidemiol Rec. 2009;40:405-20

2. World Health Organization. Guidelines for the Screening, Care and Treatment of Persons with Hepatitis C Infection. Geneva: World Health Organization; 2014. Available from: http://www.ncbi.nlm.nih.gov/books/ NBK263483/. Accessed 4 Nov 2015.

3. Gust ID. Epidemiology of hepatitis B infection in the Western Pacific and South East Asia. Gut. 1996;38 Suppl 2:S18-23.

4. Huang H, Hu XF, Zhao FH, Garland SM, Bhatla N, Qiao YL. Estimation of Cancer Burden Attributable to Infection in Asia. J Epidemiol. 2015;25:626-38.

5. Lok ASF. Chronic hepatitis B. N Engl J Med. 2002;346:1682-3. 
6. Perz JF, Armstrong GL, Farrington LA, Hutin YJF, Bell BP. The contributions of hepatitis $B$ virus and hepatitis $C$ virus infections to cirrhosis and primary liver cancer worldwide. J Hepatol. 2006;45:529-38.

7. Yu MC, Tong MJ, Coursaget P, Ross RK, Govindarajan S, Henderson BE. Prevalence of hepatitis B and $C$ viral markers in black and white patients with hepatocellular carcinoma in the United States. J Natl Cancer Inst. 1990;82:1038-41.

8. Stroffolini T, Chiaramonte M, Tiribelli C, Villa E, Simonetti RG, Rapicetta M, et al. Hepatitis C virus infection, HBsAg carrier state and hepatocellular carcinoma: relative risk and population attributable risk from a case-control study in Italy. J Hepatol. 1992;16:360-3.

9. Hadziyannis S, Tabor E, Kaklamani E, Tzonou A, Stuver S, Tassopoulos N, et al. A case-control study of hepatitis B and $C$ virus infections in the etiology of hepatocellular carcinoma. Int J Cancer J. 1995;60:627-31.

10. Iloeje UH, Yang HI, Jen CL, Su J, Wang LY, You SL, et al. Risk of pancreatic cancer in chronic hepatitis B virus infection: data from the REVEAL-HBV cohort study. Liver Int. 2010;30:423-9.

11. Fwu CW, Chien YC, Nelson KE, Kirk GD, You SL, Kuo HS, et al. Mortality after chronic hepatitis B virus infection: a linkage study involving 2 million parous women from Taiwan. J Infect Dis. 2010;201:1016-23.

12. Engels EA, Cho ER, Jee SH. Hepatitis B virus infection and risk of non-Hodgkin lymphoma in South Korea: a cohort study. Lancet Oncol. 2010;1 1:827-34.

13. Fwu CW, Chien YC, You SL, Nelson KE, Kirk GD, Kuo HS, et al. Hepatitis B virus infection and risk of intrahepatic cholangiocarcinoma and non-Hodgkin lymphoma: a cohort study of parous women in Taiwan. Hepatol Baltim Md. 2011:53:1217-25.

14. Ulcickas Yood M, Quesenberry CP, Guo D, Caldwell C, Wells K, Shan J, et al. Incidence of non-Hodgkin's lymphoma among individuals with chronic hepatitis B virus infection. Hepatol Baltim Md. 2007;46:107-12.

15. Amin J, Dore GJ, O'Connell DL, Bartlett M, Tracey E, Kaldor JM, et al. Cancer incidence in people with hepatitis B or C infection: a large communitybased linkage study. J Hepatol. 2006:45:197-203.

16. Sundquist K, Sundquist J, Ji J. Risk of hepatocellular carcinoma and cancers at other sites among patients diagnosed with chronic hepatitis B virus infection in Sweden. J Med Virol. 2014;86:18-22.

17. Hsing AW, Zhang M, Rashid A, McGlynn KA, Wang BS, Niwa S, et al. Hepatitis $B$ and $C$ virus infection and the risk of biliary tract cancer: a population-based study in China. Int J Cancer. 2008;122:1849-53.

18. Patel BB, Lipka S, Shen H, Davis-Yadley AH, Viswanathan P. Establishing the link between hepatitis B virus infection and colorectal adenoma. J Gastrointest Oncol. 2015;6:492-7.

19. Tsai MC, Kee KM, Chen YD, Lin LC, Tsai LS, Chen HH, et al. Excess mortality of hepatocellular carcinoma and morbidity of liver cirrhosis and hepatitis in HCV-endemic areas in an HBV-endemic country: geographic variations among 502 villages in southern Taiwan. J Gastroenterol Hepatol. 2007;22:92-8.

20. Liang SH, Chen TJ, Lee SSJ, Tseng FC, Huang CK, Lai CH, et al. Risk factors of isolated antibody against core antigen of hepatitis $B$ virus: association with HIV infection and age but not hepatitis C virus infection. J Acquir Immune Defic Syndr. 2010;54:122-8

21. Plummer M, de Martel C, Vignat J, Ferlay J, Bray F, Franceschi S. Global burden of cancers attributable to infections in 2012: a synthetic analysis. Lancet Glob Health. 2016;4:e609-16.

22. Shi J, Zhu L, Liu S, Xie WF. A meta-analysis of case-control studies on the combined effect of hepatitis $B$ and $C$ virus infections in causing hepatocellular carcinoma in China. Br J Cancer. 2005;92:607-12.

23. Zhang JY, Dai M, Wang X, Lu WQ, Li DS, Zhang MX, et al. A case-control study of hepatitis $B$ and $C$ virus infection as risk factors for hepatocellular carcinoma in Henan, China. Int J Epidemiol. 1998;27:574-8.

24. Donato F, Boffetta P, Puoti M. A meta-analysis of epidemiological studies on the combined effect of hepatitis B and $C$ virus infections in causing hepatocellular carcinoma. Int J Cancer J. 1998;75:347-54.

25. Blum HE, Moradpour D. Viral pathogenesis of hepatocellular carcinoma. J Gastroenterol Hepatol. 2002;17 Suppl 3:413-20.

26. Xu JH, Fu JJ, Wang XL, Zhu JY, Ye XH, Chen SD. Hepatitis B or C viral infection and risk of pancreatic cancer: a meta-analysis of observational studies. World J Gastroenterol. 2013;19:4234-41.

27. Fiorino S, Chili E, Bacchi-Reggiani L, Masetti M, Deleonardi G, Grondona AG, et al. Association between hepatitis B or hepatitis $C$ virus infection and risk of pancreatic adenocarcinoma development: a systematic review and meta-analysis. Pancreatol. 2013;13:147-60.
28. Luo G, Hao NB, Hu CJ, Yong X, Lü MH, Cheng BJ, et al. HBV infection increases the risk of pancreatic cancer: a meta-analysis. Cancer Causes Control. 2013;24:529-37.

29. Rustagi T, Zarookian El, Qasba O, Diez LF. Chronic hepatitis C as a risk factor for colorectal adenoma. Int J Colorectal Dis. 2014;29:75-80.

30. Hsieh A, Kim HS, Lim SO, Yu DY, Jung G. Hepatitis B viral X protein interacts with tumor suppressor adenomatous polyposis coli to activate Wnt/3catenin signaling. Cancer Lett. 2011;300:162-72.

31. Xu F, Zhu X, Han T, You X, Liu F, Ye L, et al. The oncoprotein hepatitis B $X$-interacting protein promotes the migration of ovarian cancer cells through the upregulation of S-phase kinase-associated protein 2 by Sp1. Int J Oncol. 2014;45:255-63.

32. Mahale P, Sturgis EM, Tweardy DJ, Ariza-Heredia EJ, Torres HA. Association Between Hepatitis C Virus and Head and Neck Cancers. J Natl Cancer Inst. 2016;108:djw035.

33. Lee MH, Yang HI, Lu SN, Jen CL, You SL, Wang LY, et al. Chronic hepatitis C virus infection increases mortality from hepatic and extrahepatic diseases: a community-based long-term prospective study. J Infect Dis. 2012;206:469-77.

34. Nyberg AH, Chung JW, Shi JM, Cheetham TC, Chiang KM, Haque R, et al. Increased cancer rates in patients with chronic hepatitis $C$ : An analysis of the cancer registry in a large U.S. health maintenance organization. Vienna: 50th Annual Meeting of the European Association for the Study of the Liver; 2015

35. Allison RD, Tong $X$, Moorman AC, Ly KN, Rupp L, Xu F, et al. Increased incidence of cancer and cancer-related mortality among persons with chronic hepatitis C infection, 2006-2010. J Hepatol. 2015;63:822-8.

\section{Submit your next manuscript to BioMed Central and we will help you at every step:}

- We accept pre-submission inquiries

- Our selector tool helps you to find the most relevant journal

- We provide round the clock customer support

- Convenient online submission

- Thorough peer review

- Inclusion in PubMed and all major indexing services

- Maximum visibility for your research

Submit your manuscript at www.biomedcentral.com/submit
Biomed Central 